\title{
RENORMALIZATION ANALYSIS OF A COMPOSITE ULTRASONIC TRANSDUCER WITH A FRACTAL ARCHITECTURE
}

\author{
EBRAHEM A. ALGEHYNE* and ANTHONY J. MULHOLLAND ${ }^{\dagger}$ \\ Department of Mathematics and Statistics \\ University of Strathclyde \\ 26 Richmond Street \\ Glasgow, G1 1XH, UK \\ *ebrahem.algehyne@strath.ac.uk \\ $\dagger$ anthony.mulholland@strath.ac.uk
}

Received November 13, 2015

Revised December 20, 2016

Accepted January 15, 2017

Published April 12, 2017

\begin{abstract}
To ensure the safe operation of many safety critical structures such as nuclear plants, aircraft and oil pipelines, non-destructive imaging is employed using piezoelectric ultrasonic transducers. These sensors typically operate at a single frequency due to the restrictions imposed on their resonant behavior by the use of a single length scale in the design. To allow these transducers to transmit and receive more complex signals it would seem logical to use a range of length scales in the design so that a wide range of resonating frequencies will result. In this paper, we derive a mathematical model to predict the dynamics of an ultrasound transducer that achieves this range of length scales by adopting a fractal architecture. In fact, the device is modeled as a graph where the nodes represent segments of the piezoelectric and polymer materials. The electrical and mechanical fields that are contained within this graph are then expressed in terms of a finite element basis. The structure of the resulting discretized equations yields to a
\end{abstract}

\footnotetext{
${ }^{*}$ Corresponding author.
}

This is an Open Access article published by World Scientific Publishing Company. It is distributed under the terms of the Creative Commons Attribution 4.0 (CC-BY) License. Further distribution of this work is permitted, provided the original work is properly cited. 
renormalization methodology which is used to derive expressions for the non-dimensionalized electrical impedance and the transmission and reception sensitivities. A comparison with a standard design shows some benefits of these fractal designs.

Keywords: Piezoelectric; Polymer; Finite Element Method; Fractal; Ultrasound; Transducer; Renormalization.

\section{INTRODUCTION}

Ultrasonic transducers are devices that are used to convert energy from one form to another. ${ }^{1}$ In this context, they convert energy from its electrical form to mechanical vibrations and vice versa. ${ }^{2,3}$ These devices can act as both transmitters and receivers; they typically work by emitting a wave (which is converted from electrical energy to mechanical energy) through a medium, and then listening and interpreting the echoes of the transmitted wave (which at this point is transformed back into electrical energy from mechanical vibrations). To further improve the transmission and reception sensitivities ${ }^{4-7}$ composite structures are utilized in piezoelectric ultrasonic transducers. Many biological species such as dolphins, bats, etc. naturally produce and receive ultrasound by utilizing a wide variety of intricate geometries in their transduction "equipment"; often with resonators spread over a range of length scales. ${ }^{8-15}$ However, man-made transducers tend to employ a regular geometry on a single length scale. Due to this characteristic, man-made transducers are unable to operate over a wide range of frequencies resulting in transmission and reception sensitivities with narrow bandwidths. To produce transducers with wider bandwidths, structures with a range of geometrical components need to be considered and one such structure is a fractal. ${ }^{16-18}$ One approach to design a new transducer is to experimentally assess its operating ability, however this is very time consuming. Each device requires materials to be sought, cut to the desired shape, bonded to other components such as matching and backing layers, and is expensive and time consuming. In addition, to determine its transmission sensitivity the device has to be immersed in a water tank, input voltages of different frequencies applied, and a hydrophone placed at some distance from the transducer to monitor the output. An assessment can also be made by connecting the transducer to an electrical circuit and measuring its electrical impedance over a range of frequencies. Given the large number of variables present in any design then the use of mathematical models to assess radically new concepts such as that proposed in this paper is fully justified. Various papers have described wave propagation in fractal media for other applications. ${ }^{19-26}$ This paper will build a model of a fractal ultrasound transducer and compare this model's operational qualities with that of a standard (simple) design. In the past, a finite differences approach ${ }^{16}$ was used in the examination of this topic; an approach in which each edge of the fractal lattice was modeled as a one-dimensional piezoelectric bar with the only degree of freedom present in the plane of the lattice. Consequently, there was no allowance for other types of motion of the lattice or directions of the electric field. This was a local description of the dynamics of the individual edges which, when joined to other edges from the lattice, led to the global dynamics of the device. To account for the three-dimensional world that the device is embedded within, this paper will derive the governing equations from the general tensor equations. This framework enables the deployment of different parameterizations and a scenario where the displacement acts out of the plane of the lattice with the electric field operating within the plane of the lattice will be examined in this paper. We will use a finite element methodology and introduce new basis functions to express the wave fields within the lattice. This Galerkin approach leads to a discrete formulation that lends itself to a renormalization approach. The Sierpinski gasket will be used for the simulation of a self-similar transducer in this paper. ${ }^{27,28}$ Such an ultrasonic transducer would start with an equilateral triangle of piezoelectric crystal. This equilateral triangle is composed of four identical equilateral sub-triangles whose side length is half of the original. The first generation $(n=1)$ would be obtained by replacing the central sub-triangle by a polymer material. This process is then repeated for several generations with the removed sub-triangles from the smallest triangles being filled with a polymer (see Fig. 1). The associated graph is constructed by a process which starts from the order $n=1$ design (which consists of three piezoelectric triangles and one polymer 


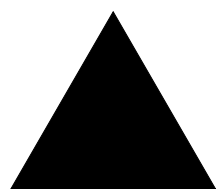

$n=0$

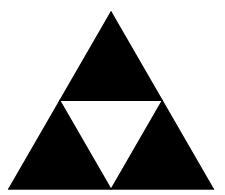

$n=1$

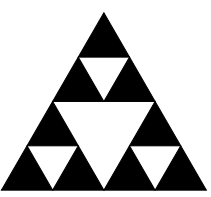

$n=2$

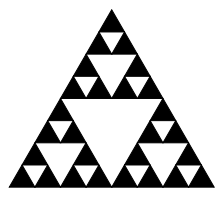

$n=3$

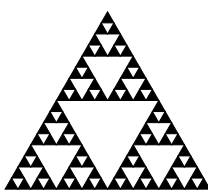

$n=4$

Fig. 1 The first few generations of the Sierpinski gasket. The black triangles are a piezoelectric material and the smallest white triangles are a polymer material.
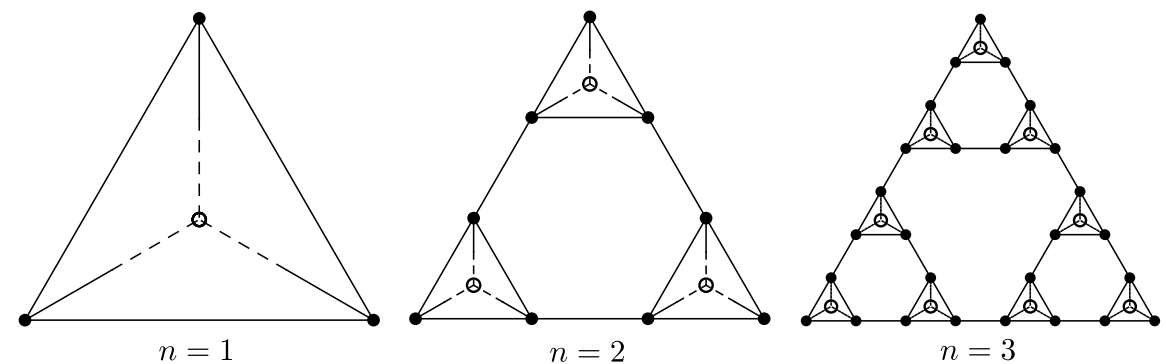

Fig. 2 The first few generations of the Sierpinski gasket lattice $\operatorname{SG}(3,4)$.

triangle), assigns a vertex to the center of each of these triangles and, by connecting these vertices together with edges, the SG(3,4) lattice at generation level $n=1$ is constructed (see Fig. 2). The polymer triangle has a vertex denoted by a nonfilled circle which was degree 3 whereas each piezoelectric triangle has a vertex denoted by a filled circle and has degree 4 . The lattice has side length $L$ units which remains constant as the generation level $n$ increases. Therefore, as $n$ increases, the length of the edge between adjacent vertices tends to zero and in this limit the lattice will perfectly match the space filling properties of the original Sierpinski gasket. ${ }^{29}$ The total number of vertices is $3^{n}+3^{n-1}$ (see Figs. 3 and 4$), h=L /\left(2^{n}-1\right)$ is the edge length between any two adjacent piezoelectric vertices, and $M=\left(5 \times 3^{n}-3\right) / 2$ denotes the total number of edges. The boundary vertices will be used to interact with external loads (both electrical and mechanical) and so we introduce fictitious vertices $A, B$ and $C$ to accommodate the interfacial boundary conditions (see Figs. 3 and 4 ). Denote by $\Omega$ the set of points lying on the edges or vertices of SG(3,4) and denote the region's boundary by $\partial \Omega$. Note that the edges joining the piezoelectric nodes to the polymer nodes are composed of a

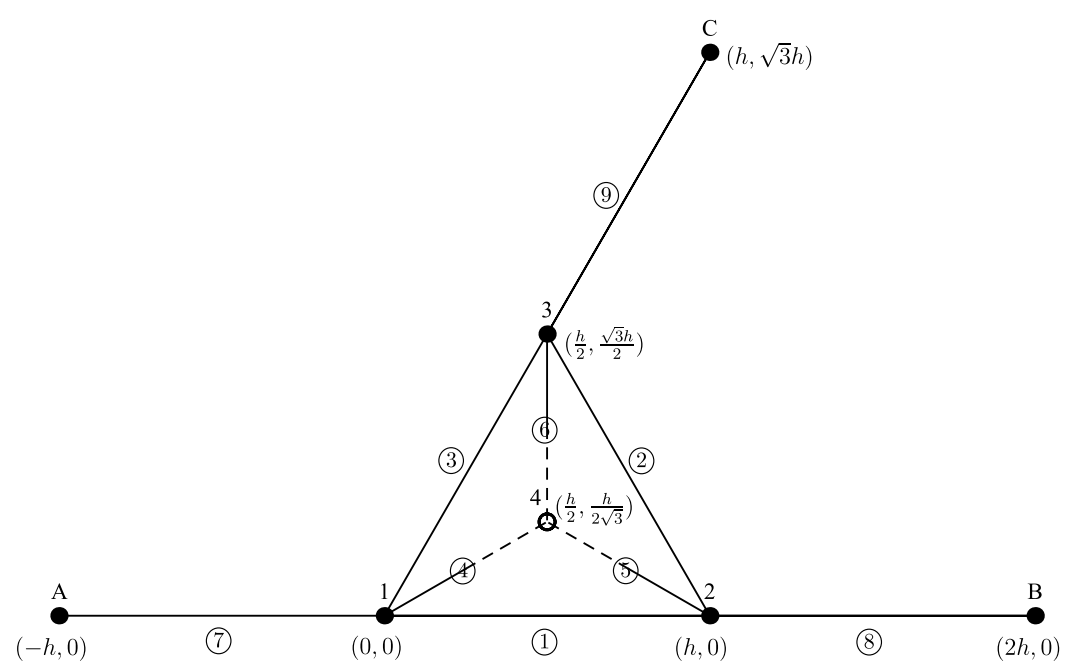

Fig. 3 The modified Sierpinski Gasket lattice $\mathrm{SG}(3,4)$ at generation level $n=1$. Nodes 1,2 and 3 are the input/output piezoelectric nodes, node 4 is a polymer node, and nodes $A$ (or 5 ), $B$ (or 6 ) and $C$ (or 7 ) are fictitious nodes used to accommodate the boundary conditions. The lattice has 9 elements (circled numbers), with two vertices adjacent to each element. 


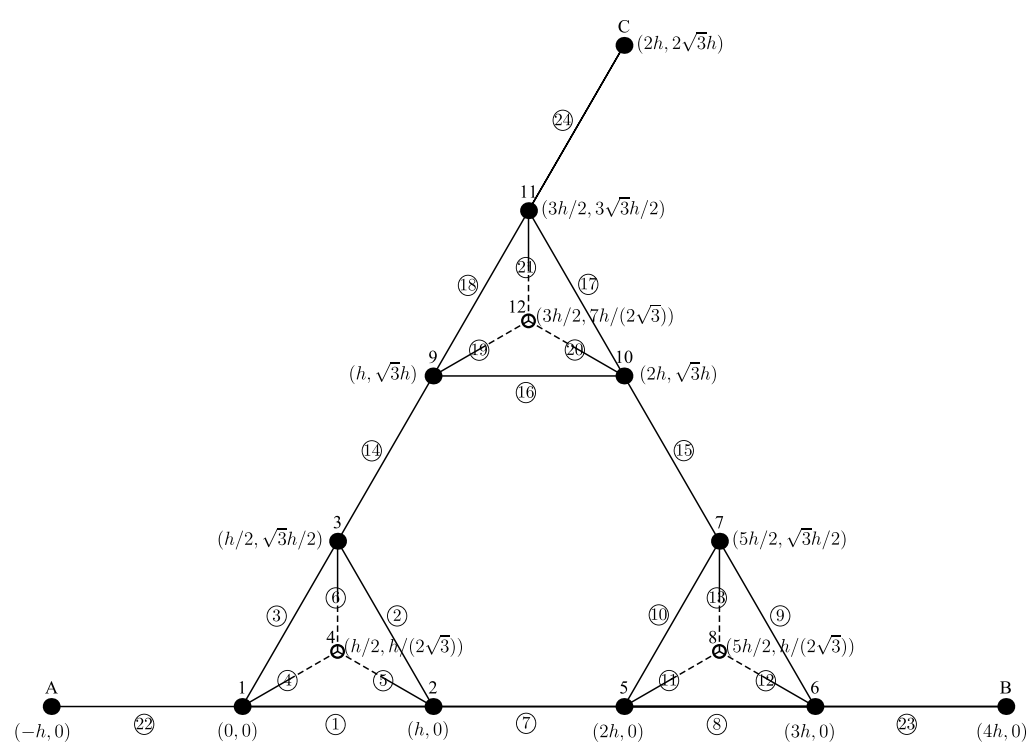

Fig. 4 The modified Sierpinski Gasket lattice $\mathrm{SG}(3,4)$ at generation level $n=2$. Nodes $A$ (or 13 ), $B$ (or 14 ) and $C$ (or 15 ) are fictitious nodes used to accommodate the boundary conditions. The lattice has 24 elements (circled numbers), with two vertices adjacent to each element.

piezoelectric section (shown by the full line in Fig. 3 along the edge joining node 1 to 4 ) and a polymer section (shown by the dashed line along this same edge). In what follows we will retain the freedom to vary the fraction of piezoelectric material in this edge from $\nu=1$ (piezoelectric material only) to $\nu=0$ (polymer material only).

\section{MODEL DERIVATION}

The lattice represents the vibrations of piezoelectric and polymer materials (here the focus will be on PZT-5H and HY1300/CY1301 hardset, ${ }^{30}$ respectively) that have been manufactured to form a Sierpinski gasket. Following a similar derivation to that in Ref. 31 we get the following equation that describes the dynamics of the displacement of the materials in the transverse direction $\left(u_{3}\right)$,

$$
\ddot{u}_{3}=c^{2} \nabla^{2} u_{3},
$$

where the dot represents differentiation with respect to time $(t)$ and $c$ is the shear wave velocity defined as

$$
c= \begin{cases}c_{T}=\sqrt{c_{44}^{T} / \rho^{E}}, & \text { PZT-5H } \\ c_{P}=\sqrt{c_{44}^{P} / \rho^{P}}, & \text { polymer }\end{cases}
$$

$\nabla^{2}=\partial^{2} / \partial x_{1}^{2}+\partial^{2} / \partial x_{2}^{2}, \quad c_{44}^{T}=c_{44}^{E}+e_{24}^{2} / \varepsilon_{11}^{E}$ is the piezoelectrically stiffened shear modulus in the ceramic phase, $c_{44}^{P}$ is the shear modulus of the polymer, $\rho^{E / P}$ is the density in the $E$ piezoelectric $/ P$ - polymer phase, $e_{24}$ is an element of the piezoelectric tensor, and $\varepsilon_{11}^{E}$ is an element of the permittivity tensor. We impose the initial conditions $u_{3}(\underline{x}, 0)=\dot{u}_{3}(\underline{x}, 0)=0$ and the boundary conditions of continuity of displacement and force on $\partial \Omega$ (the boundary to $\Omega$ ). By introducing the nondimensionalized variable $\theta=c_{T} t / h$ (temporarily dropping the subscript on $u$ and the superscript on $h)$ and applying the Laplace transform $\mathscr{L}: \theta \rightarrow q$ then Eq. (1) gives

$$
q^{2} \bar{u}=\frac{h^{2}}{c_{T}^{2}} c^{2} \nabla^{2} \bar{u} .
$$

We will seek a weak solution $\bar{u} \in H^{1}(\Omega)$ where on the boundary $\bar{u}=\bar{u}_{\partial \Omega} \in H^{1}(\partial \Omega)$. Now multiplying by a test function $w \in H_{B}^{1}(\Omega)$, where $H_{B}^{1}(\Omega):=$ $\left\{w \in H^{1}(\Omega): w=0\right.$ on $\left.\partial \Omega\right\}$, integrating over the region $\Omega$, and using Green's first identity gives

$$
q^{2} \int_{\Omega} \bar{u} w d \underline{x}=-\frac{h^{2}}{c_{T}^{2}} c^{2} \int_{\Omega} \nabla \bar{u} . \nabla w d \underline{x} .
$$

\section{GALERKIN DISCRETIZATION}

Using a standard Galerkin method we replace $H^{1}(\Omega)$ and $H_{B}^{1}(\Omega)$ by the finite dimensional subspaces $S$ and $S_{B}=S \cap H_{B}^{1}(\Omega)$. Let $U_{B} \in S$ be a function that approximates $\bar{u}_{\partial \Omega}$ on $\partial \Omega$, then the discretized problem involves finding $\bar{U} \in S$ such that

$$
q^{2} \int_{\Omega} \bar{U} W d \underline{x}=-\frac{h^{2}}{c_{T}^{2}} c^{2} \int_{\Omega} \nabla \bar{U} \cdot \nabla W d \underline{x},
$$


where $W$ is the test function expressed in this finite dimensional space. Let $\left\{\phi_{1}, \phi_{2}, \ldots, \phi_{N}, \phi_{N+1}\right\}$ form a basis of $S_{B}$ and set $W=\phi_{j}$, then

$$
\begin{array}{r}
q^{2} \int_{\Omega} \bar{U} \phi_{j} d \underline{x}=-\frac{h^{2}}{c_{T}^{2}} c^{2} \int_{\Omega} \nabla \bar{U} \cdot \nabla \phi_{j} d \underline{x}, \\
j=1, \ldots, N+1 .
\end{array}
$$

Furthermore, let $\psi_{I}, I=\{N+2, N+3, N+4\}$ form a basis for the boundary nodes and let

$$
\bar{U}=\sum_{i=1}^{N+1} U_{i} \phi_{i}+\sum_{i \in I} U_{B_{i}} \psi_{i} .
$$

Hence, Eq. (3) can be written as

$$
A_{j i}^{(n)} U_{i}=b_{j}^{(n)}
$$

where

$$
\begin{aligned}
b_{j}^{(n)}= & -\sum_{i \in I}\left(q^{2} \int_{\Omega} \psi_{i} \phi_{j} d \underline{x}\right. \\
& \left.+\frac{h^{2}}{c_{T}^{2}} c^{2} \int_{\Omega} \nabla \psi_{i} . \nabla \phi_{j} d \underline{x}\right) U_{B_{i}}, \\
A_{j i}^{(n)}= & q^{2} H_{j i}^{(n)}+\frac{h^{2}}{c_{T}^{2}} K_{j i}^{(n)}, \\
H_{j i}^{(n)}= & \int_{\Omega}\left(\phi_{j} \phi_{i}\right) d \underline{x},
\end{aligned}
$$

and

$$
K_{j i}^{(n)}=c^{2} \int_{\Omega}\left(\nabla \phi_{j} . \nabla \phi_{i}\right) d \underline{x} .
$$

\subsection{Transformations of the Fundamental Basis Functions}

In this section, we will consider transformations of some fundamental basis functions $\hat{\phi}_{J}, \hat{\phi}_{K}$ and $\hat{\psi}_{I}$ to get basis functions $\phi_{J}, \phi_{K}$ and $\psi_{I}$ at each vertex in the lattice (for the interior piezoelectric vertices $(J)$, for the interior polymer vertices $(K)$ and for the exterior piezoelectric vertices $(I))$. We choose the design of the fundamental basis functions $\hat{\phi}_{J}$ with nodes $(\sqrt{3} h / 2, h / 2),(\sqrt{3} h / 2,-h / 2),(h / \sqrt{3}, 0)$ and $(-\sqrt{3} h / 2, h / 2)$ such that (we ease the notation by setting $x_{1}=x$, and $x_{2}=y$ )

$$
\begin{aligned}
& \hat{\phi}_{j}(x, y) \\
& \quad= \begin{cases}1 & \text { if }(x, y)=\left(x_{j}, y_{j}\right) \\
0 & \text { if }(x, y)=\text { coordinates of vertices } \\
& \text { adjacent to vertex } j .\end{cases}
\end{aligned}
$$

The basis functions have a compact support and are identically zero outside the edges that are incident upon the particular vertex. For the fundamental basis functions $\hat{\phi}_{J}$ we have five nodes and so a second-order polynomial in $x$ and $y$ is chosen. By applying Eq. (10), we get

$$
\hat{\phi}_{J}(x, y)=1-\frac{3}{h^{2}} x^{2}+\frac{5}{h^{2}} y^{2} .
$$

Similarly, for the fundamental basis function $\hat{\phi}_{K}$, we get

$$
\hat{\phi}_{K}(x, y)=\frac{3}{h} x+\frac{\sqrt{3}}{h} y-\frac{3}{h^{2}}\left(x^{2}+y^{2}\right),
$$

and, for the fundamental basis functions $\hat{\psi}_{I}$ we get

$$
\hat{\psi}_{I}(x, y)=1-\frac{1}{h^{2}}\left(x^{2}+y^{2}\right) .
$$

Having established the fundamental (canonical) basis functions for each type of vertex in the lattice we now need to calculate the specific basis functions for each vertex. In order to do this each fundamental basis function is mapped onto the specific vertex by a series of transformations such as a translation, a rotation, or a reflection in the $x$ or $y$ axis (see Ref. 32 for details). The above process can then be repeated for fractal generation level $n=2$. Recall that at each generation level the overall length of the lattice remains fixed $(L)$ and the edge length $h$ decreases. As such the canonical basis functions given by Eqs. (11), (12) and (13) can still be applied here since it will be automatically scaled as its coefficients depend on $h$. For a particular element lying between vertex $i$ and vertex $j$ the isoparametric representation, given by $(x(s), y(s))=\left(\left(x_{j}-x_{i}\right) s+x_{i},\left(y_{j}-y_{i}\right) s+y_{i}\right)$ is employed, where $s_{1}=0$ and $s_{2}=1$ and $d \underline{x}=h d s$. For the elements that join a piezoelectric node to a polymer node a similar representation is used but here $d \underline{x}=h / \sqrt{3} d s$ and the region between $s=0$ and $s=\nu$ is piezoelectric and that between $s=\nu$ and $s=1$ is polymer. Substituting these into Eqs. (8) and (9), respectively, gives for each interior 
piezoelectric element $\left(e \in M_{J}\right)$,

$$
\begin{aligned}
& { }^{M_{J}} H_{j i}^{(n)} \\
& =h\left\{\begin{array}{lc}
\int_{0}^{1}\left(s^{2}-1\right)^{2} d s=\frac{16}{30} & \text { if } j=i=p, \\
\int_{0}^{1}\left(s^{2}-1\right)(s-2) s d s=\frac{11}{30} & \text { if } j, i \in\{p, q\}, \\
j \neq i, & \text { if } j=i=q, \\
\int_{0}^{1}(s-2)^{2} s^{2} d s=\frac{16}{30} & \text { otherwise, }
\end{array}\right.
\end{aligned}
$$

where element $e$ connects node $p$ to node $q$. So, for each piezoelectric - polymer element $\left(e \in M_{K}\right)$,

$$
\begin{aligned}
& { }^{M_{K}} H_{j i}^{(n)} \\
& =\frac{h}{\sqrt{3}}\left\{\begin{array}{lc}
\int_{0}^{1}\left(s^{2}-1\right)^{2} d s=\frac{16}{30} & \text { if } j=i=p, \\
\int_{0}^{1}\left(s^{2}-1\right)(s-2) s d s=\frac{11}{30} & \text { if } j, i \in\{p, q\}, \\
j \neq i, & \text { if } j=i=q, \\
\int_{0}^{1}(s-2)^{2} s^{2} d s=\frac{16}{30} & \text { otherwise. }
\end{array}\right.
\end{aligned}
$$

Note that from Eq. (7) since ${ }^{M_{K}} H_{j i}^{(n)}=h / \sqrt{3}\left(\int_{0}^{\nu}\right.$ $\left.\phi_{j} \phi_{i} d x+\int_{\nu}^{1} \phi_{j} \phi_{i} d x\right)=h / \sqrt{3} \int_{0}^{1} \phi_{j} \phi_{i} d x$, then $\nu$ does not explicitly appear. We will see later that for $c^{2} K_{j i}^{(n)}$ for $e \in M_{K}$, we need to apply Eq. (2) where ${ }^{M_{K}} c^{2} K_{j i}^{(n)}=h / \sqrt{3}\left(c_{T}^{2} \int_{0}^{\nu} \nabla \phi_{j} . \nabla \phi_{i} d s+c_{P}^{2} \int_{\nu}^{1}\right.$ $\left.\nabla \phi_{j} . \nabla \phi_{i} d s\right)$ and so $\nu$ does appear explicitly in that case. Similarly, for each exterior piezoelectric element $\left(e \in M_{I}\right)$,

$$
\begin{aligned}
& { }^{M_{I}} H_{j i}^{(n)} \\
& =h \begin{cases}\int_{0}^{1}\left(s^{2}-1\right)^{2} d s=\frac{16}{30} & \text { if } j=i=q, \\
0 & \text { otherwise, }\end{cases}
\end{aligned}
$$

where $q$ is the corner vertex of the $\mathrm{SG}(3,4)$ lattice connected to element $e$ (for $n=1, q \in\{1,2,3\}$, and for $n=2, q \in\{1,6,11\})$. Note that there is only one combination of basis functions in these exterior piezoelectric elements since the left hand side of Eq. (5) does not involve the basis functions at boundary vertices $I$ denoted by $\psi_{I}$. Eqs. (14),
(15) and (16) can then be used to assemble the full matrix in Eq. (8). Similarly, for ${ }^{e} K_{j i}^{(n)}$, for $e \in M_{J}$, using the definition of $c$ in Eq. (2), Eq. (9) leads to

$$
=\frac{2}{h} c_{T}^{2}\left\{\begin{array}{lc}
26 \int_{0}^{1} s^{2} d s=\frac{26}{3} & \text { if } j=i=p, \\
-22 \int_{0}^{1} s(s-1) d s=\frac{11}{3} & \text { if } j, i \in\{p, q\}, \\
& j \neq i, \\
26 \int_{0}^{1}(s-1)^{2} d s=\frac{26}{3} & \text { if } j=i=q, \\
0 & \text { otherwise. }
\end{array}\right.
$$

For $e \in M_{K}$,

$$
=\frac{2}{3 h} c_{T}^{M_{K}} K_{j i}^{(n)}\left\{\begin{array}{cc}
2 \sqrt{3}\left(\nu^{3}+\frac{c_{P}^{2}}{c_{T}^{2}}\left(1-\nu^{3}\right)\right) & \text { if } j=i=p, \\
\sqrt{3}\left(\nu^{2}(2 \nu-3)-\frac{c_{P}^{2}}{c_{T}^{2}}\right. & \\
\left.(\nu-1)^{2}(1+2 \nu)\right) & \text { if } j, i \in\{p, q\}, \\
2 \sqrt{3}\left(\nu\left(\nu^{2}-3 \nu+3\right)\right. & j \neq i, \\
\left.-\frac{c_{P}^{2}}{c_{T}^{2}}(\nu-1)^{3}\right) & \text { if } j=i=q, \\
0 & \text { otherwise. }
\end{array}\right.
$$

For $e \in M_{I}$,

$$
\begin{aligned}
& { }^{M_{I}} K_{j i}^{(n)} \\
& =\frac{2}{h} c_{T}^{2} \begin{cases}26 \int_{0}^{1} s^{2} d s=\frac{26}{3} & \text { if } j=i=q, \\
0 & \text { otherwise, }\end{cases}
\end{aligned}
$$

where $q$ is the corner vertex of the $\mathrm{SG}(3,4)$ lattice connected to element $e$. Combining Eqs. (14), (15), (16), (17), (18) and (19) gives Eq. (7) as

$$
A_{j i}^{(1)}=h\left[\begin{array}{llll}
\alpha & \beta & \beta & \mathcal{P} \\
\beta & \alpha & \beta & \mathcal{P} \\
\beta & \beta & \alpha & \mathcal{P} \\
\mathcal{P} & \mathcal{P} & \mathcal{P} & \vartheta
\end{array}\right]=h \hat{A}_{j i}^{(1)},
$$


where $\alpha=\left(q^{2} / 30\right)(48+(16 / \sqrt{3}))+(2 / 3)(78+$ $\left.2 \sqrt{3}\left(\nu^{3}+\left(c_{P}^{2} / c_{T}^{2}\right)\left(1-\nu^{3}\right)\right)\right), \beta=(11 / 30) q^{2}+22 / 3$, $\mathcal{P}=\left(q^{2} / 30\right)(11 / \sqrt{3})+(2 / 3)\left(\sqrt{3}\left(\nu^{2}(2 \nu-3)-\right.\right.$ $\left.\left.\left(c_{P}^{2} / c_{T}^{2}\right)(\nu-1)^{2}(1+2 \nu)\right)\right)$, and $\vartheta=\left(q^{2} / 30\right)(48 / \sqrt{3})+$ $(2 / 3)\left(6 \sqrt{3}\left(\nu\left(\nu^{2}-3 \nu+3\right)-\left(c_{P}^{2} / c_{T}^{2}\right)(\nu-1)^{3}\right)\right)$. In general $\hat{A}_{j i}^{(n)}=\bar{A}_{j i}^{(n-1)}+\beta V_{j i}^{(n)}$, where $\bar{A}_{j i}^{(n-1)}$ is a block diagonal matrix consisting of three copies of $\hat{A}_{j i}^{(n-1)}$ and $V_{j i}^{(n)}$ is the adjacency matrix for the subgraph of $\mathrm{SG}^{(n)}(3,4)$ consisting of the edges that connect each of the three $\mathrm{SG}^{(n-1)}(3,4)$ graphs. A similar treatment can be given to Eq. (6) to give (with $m=(N+1) / 2)$

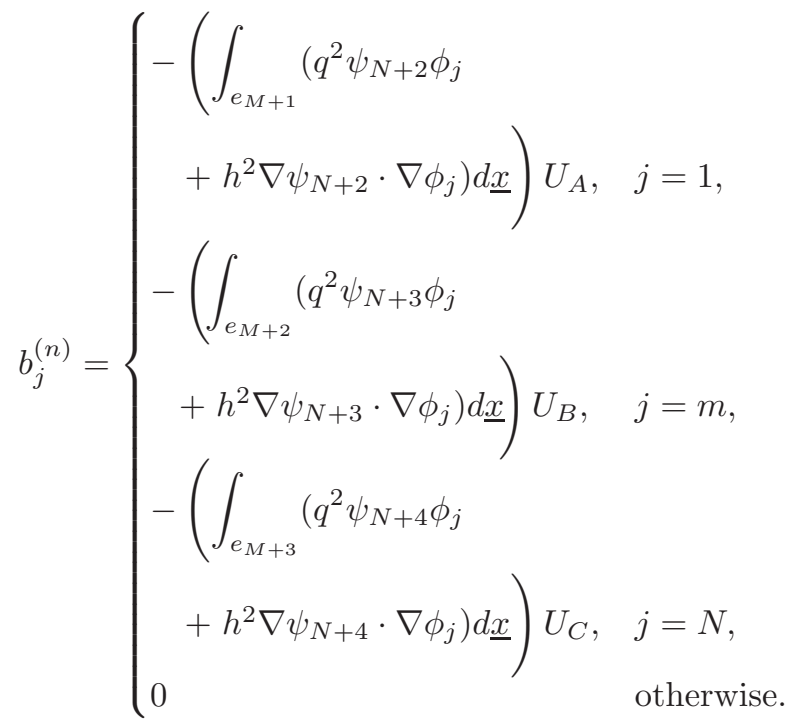

Using the isoparametric representation

$$
b_{j}^{(n)}=h \eta \begin{cases}U_{A}, & j=1, \\ U_{B}, & j=m, \\ U_{C}, & j=N, \\ 0 & \text { otherwise }\end{cases}
$$

where

$$
\eta=\frac{2}{3}-\frac{11}{30} q^{2}
$$

\subsection{Application of the Mechanical Boundary Conditions}

Mechanical and electrical loads are now introduced to the transducer at its boundaries. It can be shown that, ${ }^{32} U_{A}=\gamma_{1} U_{1}+\delta_{1}$ and $U_{B}=\gamma_{m} U_{m}+\delta_{m}=$ $U_{C}=\gamma_{N} U_{N}+\delta_{N}$, where

$$
\gamma_{j}=\left\{\begin{array}{l}
\left(1-q \frac{Z_{B}}{\overline{\bar{Z}}_{T}}\right)^{-1}, \quad j=1, \\
\left(1-q \frac{Z_{L}}{\overline{\bar{Z}}_{T}}\right)^{-1}, \quad j=m \text { or } N,
\end{array}\right.
$$

and

$$
\delta_{j}= \begin{cases}-\frac{\bar{\zeta} \bar{Q}}{\bar{c}_{44}^{T} \xi}\left(1-q \frac{Z_{B}}{\overline{\bar{Z}}_{T}}\right)^{-1}, & j=1, \\ \left(1-q \frac{Z_{L}}{\overline{\bar{Z}}_{T}}\right)^{-1} & \\ \times\left(\frac{\bar{\zeta} \bar{Q}}{\bar{c}_{44}^{T} \xi}-2 A_{L} q \frac{Z_{L}}{\overline{\bar{Z}}_{T}}\right), & j=m \text { or } N,\end{cases}
$$

where $\bar{\zeta}=\bar{e}_{24} / \bar{\varepsilon}_{11}$ and $\bar{c}_{44}^{T}$ is the effective shear modulus of the piezoelectric - polymer composite [see Eq. (42) for details]. $\bar{Q}$ is the electrical charge (in the Laplace transform domain) applied to the boundary of the transducer, $A_{L}$ is the amplitude of the incoming wave that is received by the transducer (in transmission mode $A_{L}$ is zero), the mechanical impedance of the load is $Z_{L}=A_{r} Y_{L} / c_{L}$, of the backing material is $Z_{B}=\rho_{B} c_{B} A_{r}$, and of the transducer is $\bar{Z}_{T}=A_{r} \bar{c}_{44}^{T} / c_{T}$, where $Y_{L}$ is the shear modulus, $\rho_{B}$ is the density, $c_{L}\left(c_{B}\right)$ is the wave velocity in the load (backing material) and $c_{T}$ the wave velocity in the $\mathrm{SG}^{(n)}(3,4)$ lattice. At each generation level of the Sierpinski gasket transducer the ratio of the cross-sectional area $\left(A_{r}\right)$ of each edge to its length is denoted by $\xi=A_{r} / h$. The overall extent of the lattice $(L)$ is fixed and so the length of the edges will steadily decrease and, by fixing $\xi$, the cross-sectional area will also decrease as the fractal generation level increases (in fact $A_{r}=\xi L /\left(2^{n}-1\right)$ ). Hence, Eq. (21) becomes

$$
b_{j}^{(n)}=h \bar{\gamma}_{j} U_{j}+h \bar{\delta}_{j}, \quad j=1, m \text { or } N,
$$

where $\bar{\gamma}_{j}=\eta \gamma_{j}$ and $\bar{\delta}_{j}=\eta \delta_{j}$. Putting Eq. (22) into Eq. (5) gives

$$
\hat{A}_{j i}^{(n)} U_{i}=\bar{\gamma}_{j} U_{j}+\bar{\delta}_{j},
$$

where $\hat{A}=A / h$ as in Eq. (20). Hence,

$$
\left(\hat{A}_{j i}^{(n)}-\hat{B}_{j i}^{(n)}\right) U_{i}=\bar{\delta}_{j}, \quad i=1, m \text { or } N
$$

and so

$$
U_{i}=G_{j i}^{(n)} \bar{\delta}_{j},
$$

where

$$
G_{j i}^{(n)}=\left(\hat{A}_{j i}^{(n)}-\hat{B}_{j i}^{(n)}\right)^{-1},
$$

represents the Green's transfer matrix. 


\section{RENORMALIZATION}

From Eq. (23) the desired basis function weightings in Eq. (4) at each vertex in $\Omega$ are then given by

$$
U_{j}^{(n)}=G_{j 1}^{(n)} \bar{\delta}_{1}+G_{j m}^{(n)} \bar{\delta}_{m}+G_{j N}^{(n)} \bar{\delta}_{N} .
$$

Only $U_{1}^{(n)}, U_{m}^{(n)}$ and $U_{N}^{(n)}$ will be required and so only the pivotal Green's functions $G_{i j}^{(n)}, \quad i, j \in$ $\{1, m, N\}$ need to be calculated. Temporarily ignoring matrix $\hat{B}$ (this matrix originates from consideration of the boundary conditions) then, due to the symmetries of the $\operatorname{SG}(3,4)$ lattice (and hence in matrix $\left.A^{(n)}\right), \hat{G}_{i i}^{(n)}=\hat{G}_{j j}^{(n)}=\hat{x}$, say, where $i, j \in\{1, m, N\}$ (that is, corner-to-samecorner), and $\hat{G}_{j k}^{(n)}=\hat{G}_{h k}^{(n)}=\hat{y}$, say, where $j, k, h \in$ $\{1, m, N\}, j \neq k \neq h$ (that is, corner-to-othercorner), where

$$
\hat{G}^{(n)}=\left(\hat{A}^{(n)}\right)^{-1} .
$$

At level $n+1$, we denote, $\hat{X}=\hat{G}_{i i}^{(n+1)}$ and $\hat{Y}=$ $\hat{G}_{i j}^{(n+1)}$ where $i, j, \in\{1, m, N\}, i \neq j$. An iterative procedure can be developed from Eq. (7) to give ${ }^{32}$

$$
G^{(n)}=\hat{G}^{(n)}+\hat{G}^{(n)} \hat{B}^{(n)} G^{(n)} .
$$

This system of linear equation will create the renormalization recursion relationships for the pivotal Green's functions. It will transpire that the recursions in Eq. (24) only involve two pivotal Green's functions, namely, corner-to-corner and corner-tosame-corner; the so called input/output nodes. Solving these (for $\hat{y} \neq 0, \beta \neq 0$ ) gives

$$
\hat{X}=\hat{x}+\frac{2 \beta^{2} \hat{y}^{2}\left(\hat{x}+\beta \hat{x}^{2}-\beta \hat{y}^{2}\right)}{(1+\beta \hat{x}+\beta \hat{y})\left(1-\beta^{2} \hat{x}^{2}-\beta \hat{y}+\beta^{2} \hat{y}^{2}\right)},
$$

and

$$
\hat{Y}=\frac{-\beta \hat{y}^{2}(1+\beta \hat{x}-\beta \hat{y})}{(1+\beta \hat{x}+\beta \hat{y})\left(1-\beta^{2} \hat{x}^{2}-\beta \hat{y}+\beta^{2} \hat{y}^{2}\right)} .
$$

The boundary conditions can now be re-introduced and lead to

$$
\begin{aligned}
x & =\frac{\hat{x}+2 \hat{y} \bar{\gamma}_{m} y}{1-\hat{x} \bar{\gamma}_{1}}, \\
y & =\frac{\hat{y}}{\left(1-\hat{x} \bar{\gamma}_{1}\right)\left(1-\bar{\gamma}_{m}(\hat{x}+\hat{y})\right)-2 \hat{y}^{2} \bar{\gamma}_{1} \bar{\gamma}_{m}}, \\
z & =\frac{\hat{x}+\hat{y} \bar{\gamma}_{1} y+\hat{y} \bar{\gamma}_{m} w}{1-\hat{x} \bar{\gamma}_{m}},
\end{aligned}
$$

and

$$
w=\frac{\hat{y}\left(1+\bar{\gamma}_{1} y\left(1+\bar{\gamma}_{m}(\hat{y}-\hat{x})\right)\right)}{\left(\hat{x} \bar{\gamma}_{m}-1+\hat{y} \bar{\gamma}_{m}\right)\left(\hat{x} \bar{\gamma}_{m}-1-\hat{y} \bar{\gamma}_{m}\right)},
$$

where $x=G_{11}^{(n)}, y=G_{1 N}^{(n)}, z=G_{N N}^{(n)}$ and $w=G_{m N}^{(n)}$. Following a similar derivation to that in Ref. 31 we get the following expression for the shear displacement amplitude at node $N$,

$$
\begin{aligned}
U_{N}^{(n)}= & -\frac{\bar{\zeta} \bar{Q} \eta}{\bar{c}_{44}^{T} \xi}\left(\left(1-q \frac{Z_{B}}{\overline{\bar{Z}}_{T}}\right)^{-1} G_{N 1}^{(n)}\right. \\
& \left.-\left(1-q \frac{Z_{L}}{\overline{\bar{Z}}_{T}}\right)^{-1}\left(G_{N m}^{(n)}+G_{N N}^{(n)}\right)\right) .
\end{aligned}
$$

The non-dimensionalized electrical impedance is then given by

$$
\begin{aligned}
\hat{Z}_{E}(f ; n)= & Z_{E} / Z_{0}=\frac{Z_{T}}{\bar{C}_{0} q c_{44}^{T} \xi Z_{0}} \\
& \times\left(1+\frac{\bar{\zeta}^{2} \bar{C}_{0} \eta}{\bar{c}_{44}^{T} \xi}\left(\sigma_{1}+\sigma_{2}\right)\right),
\end{aligned}
$$

where $Z_{E}$ is the dimensionalized electrical impedance of the $\mathrm{SG}^{(n)}(3,4)$ lattice, $Z_{0}$ is the series electrical impedance load, $\bar{C}_{0}=A_{r} \bar{\varepsilon}_{11} / L$ is the capacitance of the device [see Eq. (41) for $\bar{\varepsilon}_{11}$ details], $\sigma_{1}=\left(1-q\left(Z_{B} / \overline{\bar{Z}}_{T}\right)\right)^{-1}\left(G_{N 1}^{(n)}-G_{11}^{(n)}\right)$ and $\sigma_{2}=\left(1-q\left(Z_{L} / \overline{\bar{Z}}_{T}\right)\right)^{-1}\left(-G_{N m}^{(n)}-G_{N N}^{(n)}+2 G_{1 m}^{(n)}\right)$. The non-dimensionalized transmission sensitivity $\psi$ is given by

$$
\begin{aligned}
\psi(f ; n) & =\left(\frac{F}{\bar{V}}\right) / \bar{\zeta} \bar{C}_{0} \\
& =\frac{a Z_{L} Z_{T}}{\overline{\bar{Z}}_{T}\left(Z_{E}+b\right) c_{44}^{T} \xi \bar{C}_{0}} K^{(n)},
\end{aligned}
$$

where $F$ is the force on the boundary of the transducer, $\bar{V}$ is the voltage applied to the transducer, $a=Z_{P} /\left(Z_{0}+Z_{P}\right), b=Z_{0} Z_{P} /\left(Z_{0}+Z_{P}\right)$ where $Z_{P}$ is the parallel electrical impedance load and

$$
\begin{aligned}
K^{(n)}= & \left(1-q \frac{Z_{L}}{\overline{\bar{Z}}_{T}}\right)^{-1}\left(-\eta\left(\left(1-q \frac{Z_{B}}{\overline{\bar{Z}}_{T}}\right)^{-1} G_{m 1}^{(n)}\right.\right. \\
& \left.\left.-\left(1-q \frac{Z_{L}}{\overline{\bar{Z}}_{T}}\right)^{-1}\left(G_{m m}^{(n)}+G_{m N}^{(n)}\right)\right)+1\right) .
\end{aligned}
$$

The non-dimensionalized reception sensitivity $\phi$ is given by

$$
\begin{aligned}
\phi(f ; n)= & \left(\frac{\bar{V}}{F}\right)\left(\bar{e}_{24} L\right)=\frac{2 \bar{\zeta} \bar{e}_{24} L \eta \sigma_{2}}{\xi \bar{c}_{44}^{T}} \\
& \times\left(1-\frac{a Z_{T} \bar{\zeta}^{2} \eta\left(\sigma_{1}+\sigma_{2}\right)}{\left(Z_{E}+b\right) q c_{44}^{T} \bar{c}_{44}^{T} \xi^{2}}\right. \\
& \left.-\frac{a Z_{T}}{\left(Z_{E}+b\right) q c_{44}^{T} \xi \bar{C}_{0}}\right)^{-1} .
\end{aligned}
$$




\section{A SIMPLE MODEL OF THE CONVENTIONAL TRANSDUCER}

In this section, we introduce a simple model of this composite transducer ${ }^{5,33}$ that will be compared with the renormalization approach being developed here; this comparison being made at a low number of fractal generation levels (these are the most interesting cases as these are potentially manufacturable). The simple model described below can be thought of as the operating characteristics that one would obtain from a conventional (i.e. nonfractal) 1-3 composite transducer as illustrated in Fig. 5. The constitutive relations for the individual phases have a compact form, within the ceramic $(E)$ phase, and within the polymer $(P)$ phase. ${ }^{34-36}$ From the first piezoelectric constitutive equation $T_{i j}=c_{i j k l} S_{k l}-e_{k i j} E_{k}$, where $T_{i j}$ is the stress tensor, $c_{i j k l}$ is the stiffness tensor, $S_{k l}$ is the strain tensor, $e_{k i j}$ is the piezoelectric tensor and $E_{k}$ is the electric field vector, and due to the properties of PZT-5H (see Ref. 31), we get

$$
\begin{aligned}
& T_{5}^{E}=c_{44}^{E} u_{3,1}^{E}-e_{24} E_{1}^{E} \quad \text { and } \\
& T_{4}^{E}=c_{44}^{E} u_{3,2}^{E}-e_{24} E_{2}^{E} .
\end{aligned}
$$

Similarly, for polymer phase we get

$$
\begin{aligned}
& T_{5}^{P}=c_{44}^{P} u_{3,1}^{P}, \quad \text { and } \\
& T_{4}^{P}=c_{44}^{P} u_{3,2}^{P},
\end{aligned}
$$

since there is no piezoelectric effect in the polymer phase. From the second piezoelectric constitutive equation $D_{i}=e_{i k l} S_{k l}+\varepsilon_{i k} E_{k}$, where $D_{i}$ is the electrical displacement tensor and $\varepsilon_{i k}$ is the permittivity tensor (where the Einstein summation convention is adopted), and we get for the piezoelectric

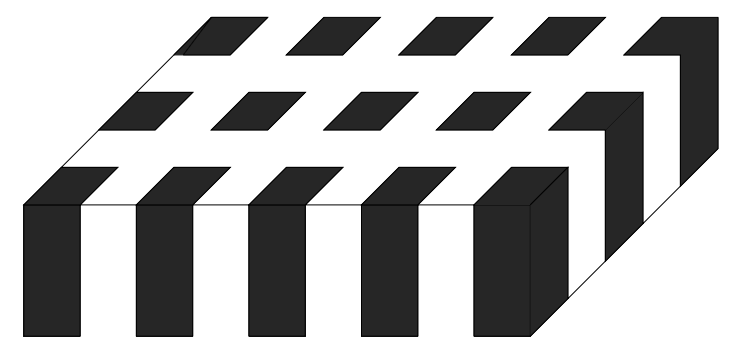

Fig. 5 Illustration of a standard 1-3 composite transducer where the ceramic is black and the polymer is white. It clearly shows the regularity in the structure and the reliance on a single length scale. phase

$$
\begin{aligned}
& D_{1}^{E}=e_{24} u_{3,1}^{E}+\varepsilon_{11}^{E} E_{1}^{E}, \quad \text { and } \\
& D_{2}^{E}=e_{24}^{E} u_{3,2}^{E}+\varepsilon_{11}^{E} E_{2}^{E},
\end{aligned}
$$

and for the polymer phase we get

$$
D_{1}^{P}=\varepsilon_{11}^{P} E_{1}^{P}, \quad \text { and } D_{2}^{P}=\varepsilon_{11}^{P} E_{2}^{P},
$$

where $D_{3}^{E}, D_{3}^{P}$ are zero. We assume that any movement (strain) in the polymer phase is compensated by a strain in the piezoelectric phase, and so we can write

$$
\begin{aligned}
& \bar{u}_{3,1}=v u_{3,1}^{E}+\bar{v} u_{3,1}^{P}, \quad \text { and } \\
& \bar{u}_{3,2}=v u_{3,2}^{E}+\bar{v} u_{3,2}^{P},
\end{aligned}
$$

where $v$ is the volume fraction of the piezoelectric phase where this is calculated via

$$
v^{(n)}=\frac{\frac{3}{2}\left(3^{n}-1\right)+3^{n-\frac{1}{2}} \nu}{\frac{3}{2}\left(3^{n}-1\right)+3^{n-\frac{1}{2}}},
$$

and $\bar{v}^{(n)}=1-v^{(n)}$ is the volume fraction of polymer. Assuming the electric fields are similarly averaged then $\bar{E}_{1}=v E_{1}^{E}+\bar{v} E_{1}^{P}$, and $\bar{E}_{2}=v E_{2}^{E}+\bar{v} E_{2}^{P}$. Assuming that the stresses in each phase are equal then $\bar{T}_{4}=T_{4}^{E}=T_{4}^{P}$ and $\bar{T}_{5}=T_{5}^{E}=T_{5}^{P}$. If the electrical displacements are also equal in each phase then $\bar{D}_{1}=D_{1}^{E}=D_{1}^{P}$ and $\bar{D}_{2}=D_{2}^{E}=D_{2}^{P}$. From the symmetry of the $\mathrm{SG}(3,4)$ lattice then we have $\bar{u}_{3,2}=\bar{u}_{3,1}=\bar{u}$, since $u_{3,2}^{E}=u_{3,1}^{E}=u^{E}$, and $u_{3,2}^{P}=$ $u_{3,1}^{P}=u^{P}$. We take the electric fields to be the same in both phases, namely, $\bar{E}_{1}=\bar{E}_{2}=\bar{E}$, since $E_{1}^{E}=$ $E_{2}^{E}=E^{E}$, and $E_{1}^{P}=E_{2}^{P}=E^{P}$. Also $\bar{T}_{4}=\bar{T}_{5}=\bar{T}$, and $\bar{D}_{1}=\bar{D}_{2}=\bar{D}$. We can then write Eq. (28) as

$$
\bar{T}=c_{44}^{E} u^{E}-e_{24} E^{E},
$$

and we can write Eq. (30) as

$$
\bar{D}=e_{24} u^{E}+\varepsilon_{11}^{E} E^{E} .
$$

For the polymer phase, we can write Eq. (29) as

$$
\bar{T}=c_{44}^{P} u^{P},
$$

and Eq. (31) as

$$
\bar{D}=\varepsilon_{11}^{P} E^{P} .
$$

We can write Eq. (32) as

$$
\bar{u}=\bar{S}=v u^{E}+\bar{v} u^{P},
$$

and we have similarly

$$
\bar{E}=v E^{E}+\bar{v} E^{P} .
$$

Hence, from Eqs. (35) and (37) we get $u^{E}=$ $\left(\bar{S}-\bar{v} \bar{T} / c_{44}^{P}\right) / v$, and from Eqs. (36) and (38) we 
get $E^{E}=\left(\bar{E}-\bar{v} \bar{D} / \varepsilon_{11}^{P}\right) / v$. Substituting these into Eq. (33) gives

$$
\bar{T}\left(1+\frac{\bar{v} c_{44}^{E}}{v c_{44}^{P}}\right)=\frac{c_{44}^{E}}{v} \bar{S}-\frac{e_{24}}{v} \bar{E}+\frac{\bar{v} e_{24}}{v \varepsilon_{11}^{P}} \bar{D},
$$

and into Eq. (34) gives

$$
\bar{D}=\frac{\varepsilon_{11}^{P} e_{24}}{\bar{\varepsilon}^{*}} \bar{S}-\frac{\bar{v} e_{24} \varepsilon_{11}^{P}}{c_{44}^{P} \bar{\varepsilon}^{*}} \bar{T}+\frac{\varepsilon_{11}^{P} \varepsilon_{11}^{E}}{\bar{\varepsilon}^{*}} \bar{E},
$$

where $\bar{\varepsilon}^{*}=v \varepsilon_{11}^{P}+\bar{v} \varepsilon_{11}^{E}$. Putting this into Eq. (39) gives

$$
\bar{T}=\bar{c}_{44} \bar{S}-\bar{e}_{24} \bar{E},
$$

where $\bar{c}_{44}=\left(c_{44}^{E} c_{44}^{P} \bar{\varepsilon}^{*}+\bar{v} c_{44}^{P} e_{24}^{2}\right) /\left(v c_{44}^{P} \bar{\varepsilon}^{*}+\bar{v} c_{44}^{E} \bar{\varepsilon}^{*}+\right.$ $\left.\bar{v}^{2} e_{24}^{2}\right)$ and $\bar{e}_{24}=\left(c_{44}^{P} e_{24} v \varepsilon_{11}^{P}\right) /\left(v c_{44}^{P} \bar{\varepsilon}^{*}+\bar{v} c_{44}^{E} \bar{\varepsilon}^{*}+\right.$ $\left.\bar{v}^{2} e_{24}^{2}\right)$. Substituting this into Eq. (40) gives $\bar{D}=\bar{e}_{24} \bar{S}+\bar{\varepsilon}_{11} \bar{E}$, where $\bar{\varepsilon}_{11}=\left(\varepsilon_{11}^{P} \varepsilon_{11}^{E}\right) / \bar{\varepsilon}^{*}+$ $\left(\bar{v} e_{24} \varepsilon_{11}^{P} \bar{e}_{24}\right) /\left(c_{44}^{P} \bar{\varepsilon}^{*}\right)$. We then have $\bar{E}=\bar{D} / \bar{\varepsilon}_{11}-$ $\bar{e}_{24} \bar{S} / \bar{\varepsilon}_{11}$, and so we can rewrite Eq. (41) as

$$
\bar{T}=\bar{c}_{44}^{T} \bar{S}-\bar{\zeta} \bar{D},
$$

where $\bar{c}_{44}^{T}=\bar{c}_{44}+\bar{e}_{24}^{2} / \bar{\varepsilon}_{11}$. The specific acoustic impedance of the composite is then, ${ }^{33}$

$$
\bar{Z}_{T}=\sqrt{\bar{c}_{44}^{T} \bar{\rho}_{T}},
$$

where $\bar{\rho}_{T}=v \rho^{E}+\bar{v} \rho^{P}$ is the average density, and the longitudinal velocity is,

$$
\bar{c}_{T}=\sqrt{\frac{\bar{c}_{44}^{T}}{\bar{\rho}_{T}}} .
$$

The current across the transducer $\bar{I}$ is then given $\mathrm{by}^{5}$

$$
\bar{I}=\frac{a \bar{V}}{Z_{E}+b} .
$$

The non-dimensionalized form for the electrical impedance of the transducer is then ${ }^{5}$

$$
\bar{Z}_{E}(f)=\frac{1}{q \bar{C}_{0} Z_{0}}\left(1-\frac{\bar{\zeta}^{2} \bar{C}_{0}}{2 q \bar{Z}_{T}}\left(\bar{K}_{F} \bar{T}_{F}+\bar{K}_{B} \bar{T}_{B}\right)\right),
$$

where $\bar{T}_{F}=2 \bar{Z}_{T} /\left(\bar{Z}_{T}+Z_{L}\right)$ and $\bar{T}_{B}=2 \bar{Z}_{T} /\left(\bar{Z}_{T}+\right.$ $\left.Z_{B}\right)$ are non-dimensional transmission coefficients, $\bar{K}_{F}$ and $\bar{K}_{B}$ are also non-dimensional and are given by

$$
\bar{K}_{F}=\frac{\left(1-e^{-q \bar{\tau}}\right)\left(1-\bar{R}_{B} e^{-q \bar{\tau}}\right)}{\left(1-\bar{R}_{F} \bar{R}_{B} e^{-2 q \bar{\tau}}\right)},
$$

and

$$
\bar{K}_{B}=\frac{\left(1-e^{-q \bar{\tau}}\right)\left(1-\bar{R}_{F} e^{-q \bar{\tau}}\right)}{\left(1-\bar{R}_{F} \bar{R}_{B} e^{-2 q \bar{\tau}}\right)},
$$

where $\bar{R}_{F}=\left(\bar{Z}_{T}-Z_{L}\right) /\left(\bar{Z}_{T}+Z_{L}\right)$ and $\bar{R}_{B}=\left(\bar{Z}_{T}-\right.$ $\left.Z_{B}\right) /\left(\bar{Z}_{T}+Z_{B}\right)$ are non-dimensionalized reflection coefficients and $\bar{\tau}=L / \bar{c}_{T}$ is the wave transit time across the device. The non-dimensionalized transmission sensitivity $\bar{\psi}$ is ${ }^{37}$

$$
\begin{aligned}
\bar{\psi}(f)= & \left(\frac{\bar{F}}{V}\right) / \bar{\zeta} \bar{C}_{0}=-\frac{a \bar{A}_{F} \bar{\lambda}_{F}}{2 \bar{C}_{0}} \\
& \times\left(1-\frac{\bar{\zeta}^{2} \bar{\lambda}\left(\bar{K}_{F} T_{F}+\bar{K}_{B} T_{B}\right)}{2 q \bar{Z}_{T}}\right)^{-1},
\end{aligned}
$$

where $\bar{\lambda}=\bar{C}_{0} /\left(1+q \bar{C}_{0} b\right)$ and $\bar{A}_{F}=$ $2 Z_{L} /\left(Z_{L}+\bar{Z}_{T}\right)$ are dimensionless constants. The non-dimensionalized reception sensitivity $\bar{\phi}$ is ${ }^{4}$

$$
\begin{aligned}
\bar{\phi}(f)= & \left(\frac{\bar{V}}{F}\right)\left(\bar{e}_{24} L\right)=\frac{-\bar{\zeta} T_{F} \bar{K}_{F} \bar{H} \bar{e}_{24} L}{q \bar{Z}_{T}} \\
& \times\left(1-\frac{\bar{\zeta}^{2} \bar{H}\left(\bar{K}_{F} T_{F}+\bar{K}_{B} T_{B}\right)}{2 q^{2} \bar{Z}_{T} Z_{E}}\right)^{-1},
\end{aligned}
$$

where $\bar{H}=q \bar{C}_{0} b /\left(1+q \bar{C}_{0} b\right)$. Having derived expressions for the main operating characteristics of a homogenized (simple) device these will be compared with the characteristics of the fractal device using the renormalization approach. This will allow us to assess any practical benefits arising from this novel design.

\section{RESULTS}

From a practical perspective, these fractal transducers will only be able to be manufactured at low fractal generation levels. For brevity we will restrict our attention to fractal generation level $n=6$. The formulation presented above will allow us to compare the fractal design (using the renormalization derivation) with a simple design (using homogenization) in terms of the key operating characteristics of the reception and transmission sensitivity spectra. ${ }^{38}$ Within each, the presence of higher amplitudes, and improved bandwidth (the range of frequencies over which the performance exceeds a certain decibel level) are the key performance indicators of interest to engineers. A typical profile of the electrical impedance spectrum (magnitude) given by Eq. (25) is shown in Fig. 6 (dashed line); it is compared to the equivalent profile given by a model of the traditional design Eq. (43) (full line). The overall trend of the curve is that of a capacitor ( $1 / f$ profile) with a prominent resonance. The important features of this plot that the design engineer is interested in are the location and magnitude of the first minimum $\left(f_{r}\right)$ and the first maximum $\left(f_{a}\right)$ turning points. The first minimum is where the mechanical resonance 


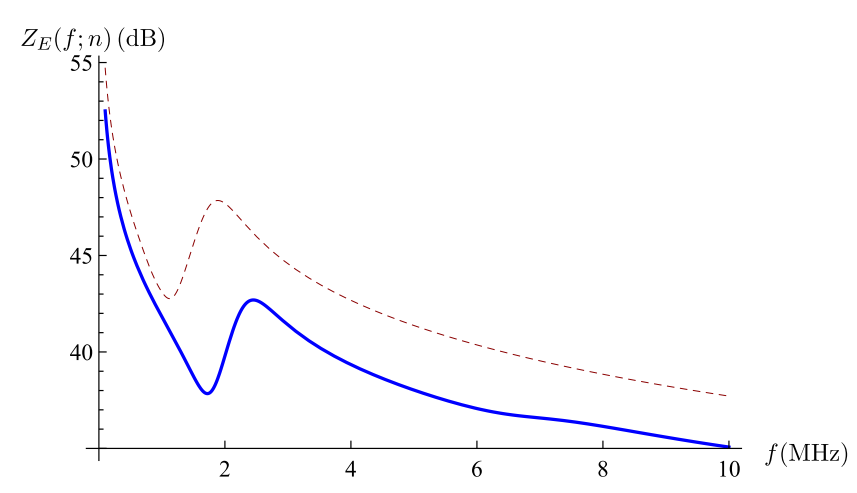

Fig. 6 Non-dimensionalized electrical impedance (Eq. (25)) versus frequency for the $\mathrm{SG}(3,4)$ lattice transducer at fractal generation level $n=6$ (dashed line). The non-dimensionalized electrical impedance of the standard (Euclidean) transducer (Eq. (43)) is plotted for comparison (full line). Parameter values are given in Table 1.

(or series resonance) occurs and, as this provides the least resistance to the electrical energy being supplied, is the frequency at which the device should be used in transmission mode. This device will produce its maximum force on the mechanical load at this frequency. The absolute value of the electrical impedance at this frequency is also important therefore and the lower it is the higher will be the peak transmission sensitivity of the device. The first maximum (known as the anti-resonance or parallel resonance frequency) is where the electrical impedance of the device peaks and is therefore the optimal frequency to operate the device in reception mode. As can be seen in Fig. 6 for the traditional design (full line) $f_{r}^{(6)}=1.7 \mathrm{MHz},\left|Z_{E}\left(f_{r} ; 6\right)\right|=38 \mathrm{~dB}$ and $f_{a}^{(6)}=2.5 \mathrm{MHz}$. As discussed above, these

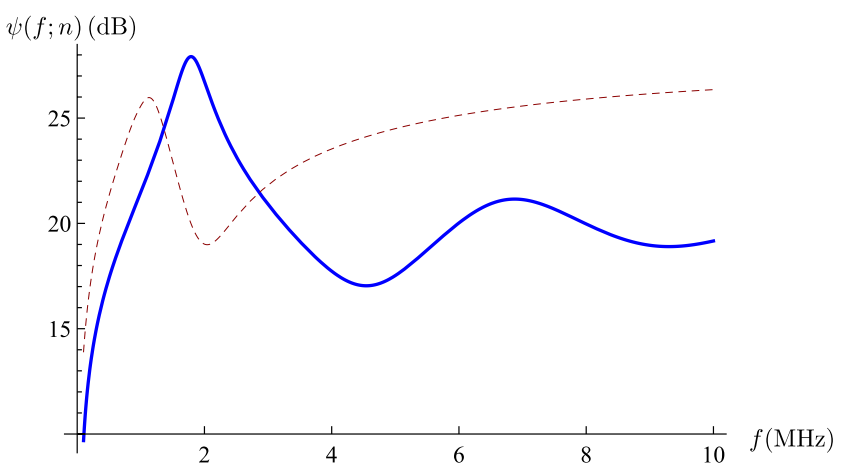

Fig. 7 Non-dimensionalized transmission sensitivity (Eq. (26)) versus frequency for the $\mathrm{SG}(3,4)$ lattice transducer at fractal generation level $n=6$ (dashed line). The nondimensionalized transmission sensitivity of the standard (Euclidean) transducer (Eq. (44)) is plotted for comparison (full line). Parameter values are given in Table 1.

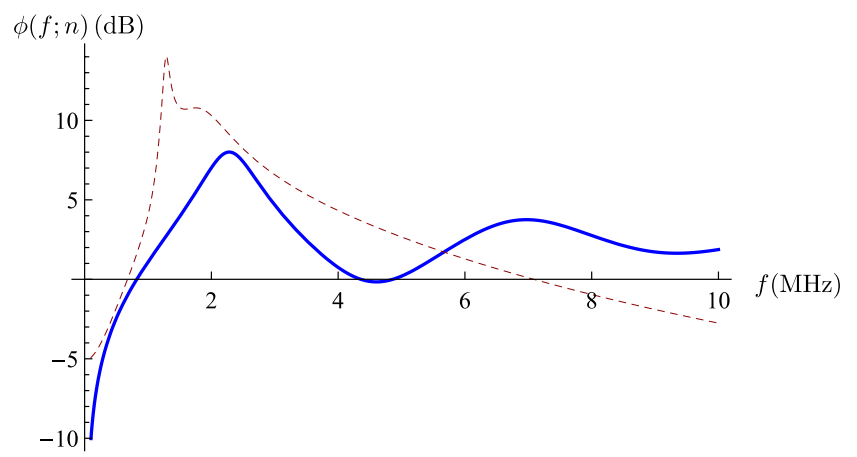

Fig. 8 Non-dimensionalized reception sensitivity (Eq. (27)) versus frequency for the $\mathrm{SG}(3,4)$ lattice transducer at fractal generation level $n=6$ (dashed line). The non-dimensionalized reception sensitivity of the standard (Euclidean) transducer (Eq. (45)) is plotted for comparison (full line). Parameter values are given in Table 1.

frequencies correspond precisely to the first maximum in the transmission sensitivity plots (Fig. 7, full line) and the reception sensitivity plots (Fig. 8, full line). From the parameter values for PZT5-H (see Ref. 39) then in Eq. (2) the piezoelectrically stiffened velocity $\left(c_{T}\right)$ is approximately $2370 \mathrm{~m} / \mathrm{s}$ and the polymer stiffened velocity $\left(c_{P}\right)$ is approximately $992 \mathrm{~m} / \mathrm{s}$ and, with an overall device length of $L=0.5 \mathrm{~mm}$, then the first mechanical resonant frequency is approximately $f_{a}=c_{T} /(2 L)=2.4 \mathrm{MHz}$. This agrees reasonably well with the reception sensitivity maximum for the homogenized (simple) estimate for $f_{a}$. For the Sierpinski gasket design the electrical impedance resonance frequency is much lower $\left(f_{r}^{(6)}=1.2 \mathrm{MHz}\right.$ and $\left.f_{a}^{(6)}=2 \mathrm{MHz}\right)$ and this suggests that it is a complex interaction between the edge lengths in the graph associated with the various generation levels that is causing this; so the internal geometry is dictating the device behavior as anticipated. Importantly, the magnitude of the electrical impedance at the electrical resonance frequency is higher than the traditional design; there is about a $5 \mathrm{~dB}$ increase for $n=6$. This results in the reception sensitivity spectrum having a much larger gain for $n=6$; there is a $7 \mathrm{~dB}$ improvement in the reception sensitivity gain from the traditional design to the fractal design (see Fig. 8). Importantly, this peak in the reception sensitivity also results in an enhanced bandwidth; if we take the noise floor to be $3 \mathrm{~dB}$ below the peak gain of the traditional design (that is $5 \mathrm{~dB}$ ) then the operational bandwidth of the traditional design is 1.5 $\mathrm{MHz}$ whereas the fractal design has an operational bandwidth of around $3 \mathrm{MHz}$. It should be borne in mind of course that no matching layers 
Table 1 Parameter Values for the Sierpinski Gasket Transducer. ${ }^{16,44}$

\begin{tabular}{lccc}
\hline Design Parameter & Symbol & Magnitude & Dimensions \\
\hline Parallel electrical impedance load & $Z_{P}$ & 1000 & Ohms \\
Series electrical impedance load & $Z_{0}$ & 50 & Ohms \\
Length of fractal & $L$ & 0.5 & $\mathrm{~mm}$ \\
Mechanical impedance of the front load & $Z_{L}$ & 1.5 & MRayls \\
Mechanical impedance of the backing layer & $Z_{B}$ & 0.02 & MRayls \\
\hline
\end{tabular}

(or indeed an optimized backing layer) have been used in this design, and that the transducer is solely composed of the piezoelectric-polymer composite material. The transmission sensitivity of the fractal design has a maximum amplitude (gain) that is lower than the Euclidean case (at $1.1 \mathrm{MHz}$ its sensitivity is $26 \mathrm{~dB}$ and the peak sensitivity of the standard device is $28 \mathrm{~dB}$ at $1.8 \mathrm{MHz}$ ), and the bandwidth is also smaller than that of the simple case (at the $-3 \mathrm{~dB}$ level which equates to the $25 \mathrm{~dB}$ level). Similar findings result when one studies other fractal generation levels (see Ref. 32) but to keep this paper concise we have restricted attention to $n=6$. In the work of Sapoval and co-workers, the support for the vibrations is a continuous surface with a fractal boundary. ${ }^{40}$ So the vibrations occur inside a fractal boundary. In this paper, the support for the vibration is a fractal graph. So the entire support is fractal and not just the boundary. So the vibrations occur on a fractal rather than inside one. The additional resonances that occur have been well documented in non-piezoelectric materials - see the work of Kigami ${ }^{19}$ and Strichartz. ${ }^{41}$ In this second scenario, the various length scales associated with the fractal add to the broadening of the frequency response. This has found some utility for example in the design of fractal antennae. ${ }^{42}$ Traditional ultrasonic transducer designs are based on a single length scale and so in this paper, by using the range of length scales found in fractal graphs, the bandwidth can be increased.

\section{CONCLUSIONS}

The performance of a composite piezoelectric ultrasound transducer, where its internal architecture is a fractal, is compared with that of a traditional design. The former case is modeled using a renormalization approach whereas the latter case is modeled using homogenization (simple). In a previously published paper, ${ }^{31,43}$ only ceramic elements were used, however in this paper, this was improved on by using a combination of ceramic and polymer elements. New basis functions, whose support is the underlying fractal graph, were developed for the finite element analysis. To assess the performance of this new device a model for a homogenized (simple) device was derived. This represents the standard designs that are used whereby the piezoelectric and polymer constituents are on the same length scale and are often arranged in a periodic structure. A low fractal generation level of the fractal transducer was investigated as this is in the regime most likely to be amenable to manufacture. A significantly higher amplitude reception sensitivity was produced by the fractal transducer when compared to the standard design; note however that a lower transmission sensitivity amplitude resulted. The reception sensitivity also resulted in a wider bandwidth than the standard design; if we take the noise floor to be $3 \mathrm{~dB}$ below the peak gain of the traditional design. The positive results in this theoretical work have subsequently led to a program to manufacture these fractal devices. Our future work will then focus on a comparison between the results presented here and our experimental findings.

\section{ACKNOWLEDGMENTS}

The authors gratefully acknowledge the support given by the Ministry of Education, University of Tabuk in Saudi Arabia and the Royal Embassy of Saudi Arabia in UK. This work was funded by the UK Engineering and Physical Sciences Research Council [grant number EP/P005268/1].

\section{REFERENCES}

1. R. T. Beyer and S. V. Letcher, Physical Ultrasonics (Academic Press, New York, 1969).

2. J. Yang, The Mechanics of Piezoelectric Structures (World Scientific, Singapore, 2006).

3. J. Yang, Analysis of Piezoelectric Devices (World Scientific, Singapore, 2006). 
4. G. Hayward, A systems feedback representation of piezoelectric transducer operational impedance, Ultrasonics 22 (1984) 153-162.

5. L. A. Orr, A. J. Mulholland, R. L. O'Leary, A. Parr, R. Pethrick and G. Hayward, Theoretical modelling of frequency dependent elastic loss in composite piezoelectric transducers, Ultrasonics 47(1) (2007) $130-137$.

6. A. Alippi, A. Bettucci, F. Craciun, F. Farrelly, E. Molinari and A. Petri, Acoustic modes in 2dimensional self-similar composite plates, Vol. 1, IEEE Ultrasonics Symp. (IUS), Baltimore, MA, USA, (1993), pp. 533-536.

7. E. A. Algehyne and A. J. Mulholland, Sensor mimics nature to detect dangerous structural cracks, Homeland Defense and Security Information Analysis Center (HDIAC), Spotlight website, 31 August, 2015, Available at https://www.hdiac.org/node/ 2163.

8. R. Müller, H. Lu, S. Zhang and H. Peremans, A helical biosonar scanning pattern in the Chinese Noctule Nycatalus Plancyi, JASA 119(6) (2006) 4083-4092.

9. R. Müller, A numerical study of the role of the tragus in the big brown bat, JASA, 116(6) (2004) 3701-3712.

10. R. N. Miles and R. R. Hoy, The development of a biologically-inspired directional microphone for hearing aids, Audiol. Neurootol. 11(2) (2006) 86-94.

11. A. M. Chiselev, L. Moraru and A. Gogu, Localization of an object using a bat model inspired from biology, J. Biophys. 19(4) (2009) 251-258.

12. D. F. Eberl, R. W. Hardy and M. J. Kernan, Genetically similar transduction mechanisms for touch and hearing in Drosophila, J. Neurosci. 20(16) (2000) 5981-5988.

13. B. Nadrowski, J. T. Albert and M. C. Göpfert, Transducer-based force generation explains active process in Drosophila hearing, Curr. Biol. 18 (2008) 1365-1372.

14. D. Robert and M. C. Göpfert, Novel schemes for hearing and orientation in insects, Curr. Opin. Neurobiol. 12 (2002) 715-720.

15. F. M. de Espinosa, O. Martinez, L. E. Segura and L. Gomez-Ullate, Double frequency piezoelectric transducer design for harmonic imaging purposes in NDT, IEEE. TUFFC, 52(6) (2005) 980-986.

16. A. J. Mulholland and A. J. Walker, Piezoelectric ultrasonic transducers with fractal geometry, Fractals 19(4) (2011) 469-479.

17. A. J. Mulholland, A. J. Walker, J. W. Mackersie, R. L. O'Leary, A. Gachagan and N. Ramadas, The use of fractal geometry in the design of piezoelectric ultrasonic transducers. 2011 IEEE International Ultrasonics Symp. (IUS), Orlando, Florida, USA, doi: 10.1109/ULTSYM.2011.0387:1559-1562, (2011).
18. L. A. Orr, A. J. Mulholland, R. L. O'Leary and G. Hayward, Analysis of ultrasonic transducers with fractal architecture, Fractals 16(4) (2008) 333-349.

19. J. Kigami, Analysis on Fractals (Cambridge University Press, Cambridge, UK, 2001).

20. K. Falconer and J. Hu, Nonlinear diffusion equations on unbounded fractal domains, J. Math. Anal. Appl. 256 (2001) 606-624.

21. M. Giona, Transport phenomena in fractal and heterogeneous media: Input/output renormalization and exact results, Chaos Solitons Fractals $\mathbf{7}(9)$ (1996) 1371-1396.

22. J. Abdulbake, A. J. Mulholland and J. Gomatam, A renormalization approach to reaction-diffusion processes on fractals, Fractals 11(4) (2003) 315330 .

23. J. Abdulbake, A. J. Mulholland and J. Gomatam, Existence and stability of reaction-diffusion waves on a fractal lattice, Chaos Solitons Fractals 20(4) (2004) 799-814.

24. M. T. Barlow, Diffusion on fractals. In Lectures on probability theory and statistics, ed. P. Bernard, Lecture Notes in Mathematics, Vol. 1690 (Springer, Berlin, 1998), pp. 1-121.

25. G. Derfel, P. J. Grabner and F. Vogl, Laplace operators on fractals and related functional equations, $J$. Phys. A Math. Theor. 45(46) (2012) 463001.

26. M. Giona, W. A. Schwalm, M. K. Schwalm and A. Adrover, Exact solution of linear transport equations in fractal media - I. Renormalization analysis and general theory, Chem. Eng. Sci. 51(20) (1996) 4717-4729.

27. K. Falconer, Fractal Geometry: Mathematical Foundations and Applications (John Wiley and Sons Ltd, Chichester, England, 2003).

28. W. A. Schwalm and M. K. Schwalm, Extension theory for lattice Green functions, Phys. Rev. B 37(16) (1988) 9524-9542.

29. A. J. Mulholland, Bounds on the Hausdorff dimension of a renormalisation map arising from an excitable reaction-diffusion system on a fractal lattice, Chaos Solitons Fractals 35(2) (2008) 274284.

30. R. L. O'Leary, G. Smillie, G. Hayward and A. C. S. Parr, CUE materials database, technical report, Centre for Ultrasonic Engineering, University of Strathclyde, Glasgow, Scotland (2002). www.cue.ac.uk.

31. E. A. Algehyne and A. J. Mulholland, A finite element approach to modelling fractal ultrasonic transducers, IMA J. Appl. Math. 80(6) (2015) 1684-1702.

32. E. A. Algehyne and A. J. Mulholland, A composite ultrasonic transducer with a fractal architecture, Department of Mathematics and Statistics, University of Strathclyde, Departmental report (11) (2015). 
33. W. A. Smith and B. A. Auld, Modeling 1-3 composite piezoelectric: Thickness-mode oscillations, IEEE 38(1) (1991) 40-47.

34. H. F. Tiersten, Linear Piezoelectric Plate Vibrations (Plenum, New York, 1969).

35. H. F. Tiersten, Thickness vibrations of piezoelectric plates, JASA 35(1) (1963) 53-58.

36. IEEE and ANSI, Institute of electrical and electronic engineers, inc. An American National Standard: IEEE Standard on Piezoelectricity (1988).

37. G. Hayward, C. J. McLeod and T. S. Duranni, A systems model of the thickness mode piezoelectric transducer, JASA 76(2) (1984) 369-382.

38. Wolfram Research, Inc. Mathematica. Version 10.0. Champaign, Illinois (2014).

39. B. A. Auld, Acoustic Fields and Waves in Solids, Vol. 1 (John Wiley and Sons, New York, 1973).
40. S. Russ and B. Sapoval, Increased damping of irregular resonators, Phys. Rev. E 65(3) (2002) 036614.

41. R. S. Strichartz, Analysis on fractals, Notices AMS 46(10) (1999) 1199-1208.

42. H. D. Werner, L. R. Haupt and L. P. Werner, Fractal antenna engineering: The theory and design of fractal antenna arrays, IEEE Antennas and propagation Mag. 41(5) (1999) 37-58.

43. E. Barlow, E. A. Algehyne and A. J. Mulholland, Investigating the performance of a fractal ultrasonic transducer under varying system conditions, Symmetry 8(6) (2016) 43-72.

44. A. J. Mulholland, S. N. Ramadas, R. L. O'Leary, A. C. S. Parr, A. Troge, G. Hayward and R. A. Pethrick, A theoretical analysis of a piezoelectric ultrasound device with an active matching layer, Ultrasonics 47(1) (2007) 102-110. 